\title{
Comparative Biochemical and Motor Function Analysis of Alpha Lipoic Acid and N-Acetyl Cysteine Treatment on Rats with Experimental Spinal Cord Injury
}

\author{
Ahmet Gurhan GURCAY ${ }^{1}$, Oktay GURCAN ${ }^{1}$, Atilla KAZANCl ${ }^{1}$, Ismail BOZKURT ${ }^{1}$, Salim SENTURK², \\ Ebru BODUR ${ }^{3}$, Omer Faruk TURKOGLU', Murad BAVBEK ${ }^{1}$
}

${ }^{1}$ Ankara Ataturk Research and Training Hospital, Department of Neurosurgery, Ankara, Turkey
${ }^{2}$ Koc University, Medical School Hospital, Department of Neurosurgery, Istanbul, Turkey
${ }^{3}$ Hacettepe University, Faculty of Medicine, Department of Medical Biochemistry, Ankara, Turkey

\section{ABSTRACT}

AIM: Spinal Cord Injury (SCl) is a devastating health problem both for the patient and the clinician. Numerous treatment modalities have been studied to reverse the effects of spinal cord injury. Herein is reported the effects and the comparison of Alpha Lipoic Acid and N-Acetyl Cysteine on rats with SCl.

MATERIAL and METHODS: 38 adult male Sprague-Dawley rats were randomly divided into 5 groups: only laminectomy, laminectomy and trauma, laminectomy trauma and Alpha Lipoic Acid $100 \mathrm{mg} / \mathrm{kg} \mathrm{IP}$ administration, laminectomy trauma and N-Acetyl Cysteine $300 \mathrm{mg} / \mathrm{kg}$ IP administration, and vehicle group (PEG). The trauma model was the Modified Allen Weight drop method. After the procedure, the rats' motor function was evaluated using the modified Tarlov Scale and consequently they were sacrificed and the spinal cord tissue was analyzed biochemically for inflammation markers.

RESULTS: Both Alpha Lipoic Acid and N-Acetyl Cysteine administration after the injury significantly improved the results. There was no statistically significant difference in between the agents.

CONCLUSION: Although these agents both proven to be effective in ameliorating the effects of SCl, there was not enough evidence in this research to conclude the benefit of one agent over the other.

KEYWORDS: Alpha lipoic acid, N-Acetyl Cysteine, Spinal cord injury, Secondary phase, MDA, TNF-a, IL-6

\section{INTRODUCTION}

Neuro-trauma is one of the most devastating health care and social problems in both developed and underdeveloped countries. Spinal cord injury (SCl) constitutes a major portion of neuro-traumas. Global prevalence of $\mathrm{SCl}$ varies from 236 to 1.280 per million inhabitants (6). An enormous amount of resources and manpower has been made available to reverse the effects of SCl. The processes, which have been investigated for ameliorating the results of the injury, have been the target of novel treatments.
SCl develops in two phases: primary and secondary injury. The mechanical impact of the trauma destroys neurons and disrupts the architecture of the tissue, composing the primary phase, but it also induces the overproduction of deleterious substances, which provokes the secondary injury cascade (13).

For the secondary injury cascade, numerous distinct mechanisms have been hypothesized and analyzed. The activation of microglia and oxidative stress are the pivotal secondary injury mechanisms after traumatic injury that construct the 
onset of neuro-inflammation. Both free oxygen radicals and pro-inflammatory cytokines are disproportionately secreted after $\mathrm{SCl}$. Hydrogen peroxide, superoxide anions and IL-1 $\beta$, IL-6, IL-12, and Tumor Necrosis Factor alpha (TNF-a) are some of the numerous examples (10). Lipid peroxidation of cellular membranes, oxidation of proteins and DNA fragmentation are some additional secondary injury cascades that may contribute to cellular damage $(5,12)$.

Mitochondrial dysfunction has a key role in the deleterious effects of reactive oxygen species (ROS.) Preserving the mitochondrial functions is the novel target for minimizing the harmful effect of free oxygen radical damage. Living organisms have developed various antioxidant systems against ROS throughout evolution.

Glutathione (GSH) is believed to be one of the paramount antioxidants in living organisms. It is a complex molecule that is mostly made of cysteine, glycine and glutamate. Both Alpha Lipoic Acid (a-LA) and N-Acetyl Cysteine (NAC) are thiol derivatives and take part in regenerating $\operatorname{GSH}(18,20)$. a-LA and NAC may compose an imperative element of the antioxidant systems $(15,20)$.

a-LA was identified as a coenzyme in the tricarboxylic acid cycle (Krebs cycle) in 1951 (17). a-LA has a small molecular weight. Metals such as iron and mercury are known as free radical damage mediators. a-LA not only chelates these metals but also plays a role in extinguishing ROS and restoring GSH, which is a potential antioxidant enzyme (1).

NAC is a thiol derivative, which takes part in maintaining the optimal redox levels in mitochondria. NAC is an acetylated precursor for cysteine and takes part in maintaining of bioenergetics of mitochondria and exhibiting antiinflammatory actions $(10,18)$. It has been postulated that NAC possesses anti-inflammatory activity by reducing the levels of pro-inflammatory cytokines (8). The neuroprotective effects of NAC appear to be mediated by both antioxidant and antiinflammatory effects.

Though neural tissue has limited antioxidative capacity (7), it could be postulated that administration of antioxidants such as a-LA and NAC have the potential to protect the neural tissue from deleterious ROS effects.

The goal of the present study was to compare of the neuroprotective effects of a-LA and NAC, as antioxidant agents, following administration in the secondary injury phase after traumatic spinal cord injury in rats. Levels of pro-inflammatory cytokines such as TNF-alpha, IL-6 and malondialdehyde (MDA), which is one of the end products of lipid peroxidation, are evaluated regarding the neuro-protective potential of a-LA and NAC, just after traumatic spinal cord injury is created by the weight drop model. The results reported here support the benefit of both a-LA and NAC administration for ameliorating spinal cord injury.

\section{MATERIAL and METHODS}

The study was approved by Ethic Committee of Kobay (Approval date 03/09/2012 No: 51\&53). Adult male Sprague-
Dawley rats (weighing between 250-300g, $\mathrm{n}=40$ ) were recruited in the study; 2 cases were deceased after laminectomy and thus 38 rats were used. They were kept in a temperaturecontrolled room $\left(23^{\circ} \mathrm{C}\right)$ with a 12-hour light/dark cycle (lights on at 7:00 AM) and with free access to water and food. Experiments were performed between 8:00 and 12:00 AM.

\section{Surgical Procedure}

All surgical techniques were performed under aseptic conditions. Animals were anesthetized with an intraperitoneal (IP) injection mixture of Ketamin (Ketalar, Pfizer Istanbul, Turkey) $90 \mathrm{mg} / \mathrm{kg}$ and Xylazine (Rompun, Bayer, Istanbul, Turkey) $10 \mathrm{mg} / \mathrm{kg}$. Arterial saturation, cardiac rate, and rectal temperature were monitored. The body temperature of the animals was fixed at a range of $37^{\circ} \mathrm{C} \pm 5^{\circ} \mathrm{C}$ by using a heating pad.

After shaving and cleansing of the skin, animals were kept in a stereotaxic apparatus and a posterior midline incision was performed in the mid-thoracic region. The underlying muscles were retracted and total laminectomy was performed with the help of a microscope.

After the surgical procedure, the paraspinal fascia, muscle and skin were closed with 4-0 nylon suture (Ethicon, ETHILON'M nylon sutures Reverse Cutting Size 4-0, 18" Black Monofilament Needle FS-2, 3/8 circle). The animals were placed in a heating chamber and body temperature was maintained at approximately $37^{\circ} \mathrm{C}$ until they were fully awake, at which time they were returned to their cages, 2 or 3 rats per cage. Postoperative care included regular bladder excretion.

\section{Traumatic Injury Model}

Modified Allen's weight-drop technique was used in this study (2). The force applied during the trauma was $40 \mathrm{~g}-\mathrm{cm}$. The extent of the trauma was measured by multiplying weight and height, expressed as $\mathrm{g}-\mathrm{cm}$. Briefly, a $5-\mathrm{mm}$-diameter cylindrical glass tube was positioned at a $90^{\circ}$ angle on the surface of the exposed dura mater, and a $4 \mathrm{~g}$ cylindrical constant weight was dropped from a $10-\mathrm{cm}$ height through the tube onto the spinal cord.

\section{Experimental Groups}

The animals were randomly divided into 5 groups $(n=5)$. The main characteristics of the groups are summarized in Table I.

Group 1 (L): Sham-operated animals (negative control group) (7).

In this group, a mid-thoracic skin incision was made, the para-vertebral muscles were dissected, and the laminae were exposed. Laminectomies were carried out at T6-8 as noted above without any experimental procedure. The muscles and the skin were closed with 4-0 nylon sutures.

Group 2 (LT): Trauma-only animals (positive control) (8).

In this group, laminectomies were performed and $\mathrm{SCl}$ was induced as described above. The site of injury was marked with a prolene suture at the neighboring tissue. 
Gurcay AG. et al: Alpha Lipoic Acid and N-Acetyl Cysteine in SCl

Table I: Group Details

\begin{tabular}{ll}
\hline Group & Procedure \\
\hline Group 1-L (negative control) & Only laminectomy \\
\hline Group 2-LT (positive control) & Laminectomy and trauma \\
\hline Group 3-LA (experimental) & Laminectomy and trauma, $100 \mathrm{mgr} / \mathrm{kg} \mathrm{IP,} \mathrm{a-LA} \mathrm{administered} \mathrm{immediately} \mathrm{after} \mathrm{trauma}$ \\
\hline Group 4-NAC (experimental) & Laminectomy and trauma, $300 \mathrm{mgr} / \mathrm{kg}$ IP, NAC administered immediately after trauma \\
\hline Group 5-PEG (vehicle) & Laminectomy and trauma, $0.5 \mathrm{ml}, \mathrm{IP}, \mathrm{PEG}$ administered immediately after trauma \\
\hline
\end{tabular}

IP: intraperitoneally PEG: Poly Ethylene Glycol.

Group 3 (LA): Experimental group animals (8).

In this group, after performing laminectomies and spinal trauma, rats received a-LA injection $(100 \mathrm{mg} / \mathrm{kg}$ ) intraperitoneally immediately after trauma.

Group 4 (NAC): Experimental group animals (8).

In this group, after performing laminectomies and spinal trauma, rats received NAC injection $(300 \mathrm{mg} / \mathrm{kg}$ ) intraperitoneally immediately after trauma.

Group 5 (PEG): Vehicle group animals (7).

In this group, after performing laminectomies and spinal trauma, rats received $0.5 \mathrm{ml}$ PEG (Poly Ethylene Glycol carrier for NAC and a-LA) administered immediately after trauma.

\section{Tissue preparation}

All groups were sacrificed 24 hours later after perfusion. The thorax was dissected and the left ventricle was cannulated to perform intracardiac perfusion with $100-300 \mathrm{ml}$ phosphate buffered saline $(\mathrm{pH} 7.4)$ and $100 \mathrm{ml}$ paraformaldehyde (4\% in $0.1 \mathrm{~mol}$ phosphate buffered saline) (19). All hemorrhagic components of the spinal cord tissue were removed with $\mathrm{NaCl}$ $0.9 \%$ solution. Spinal cord samples were excised from nearly cranial and caudal points of the injury level (approximately 1 $\mathrm{cm}$ of spinal cord) with the help of a microscope and micro tools and kept in liquid nitrogen until analysis.

\section{Biochemical Analysis}

\section{Lipid Peroxidation / MDA-TBARS assay}

The OxiSelect ${ }^{\mathrm{TM}}$ TBARS Assay Kit used in the measurement of lipid peroxidation (MDA) is dependent on the principle that MDA forms a conjugate bond with thiobarbituric acid with a ratio of $1: 2$. The homogenates were diluted with $5 \%$ butylated hydroxytoluene. Then, $100 \mu \mathrm{L}$ of sodium laurylsulfate (SDS) was added to $100 \mu \mathrm{L}$ of this mixture and the resultant mixture was incubated at room temperature for 5 min. Following this incubation period, $250 \mu \mathrm{L}$ of thiobarbituric acid (TBA) was added to the mixture and incubated for $45 \mathrm{~min}$ at $95^{\circ} \mathrm{C}$. The tubes were cooled over ice for $5 \mathrm{~min}$ and then centrifuged at $10,000 \times \mathrm{g}$ for $15 \mathrm{~min}$. In a new tube, $200 \mu \mathrm{L}$ of supernatant and $300 \mu \mathrm{L}$ of butyl alcohol was mixed on a vortex mixer for 3 $\mathrm{min}$ at $3000 \mathrm{rpm}$ and then centrifuged for $5 \mathrm{~min}$ at 10,000 $\mathrm{x} \mathrm{g}$. The supernatant absorbance at $532 \mathrm{~nm}$ was measured using the Molecular Devices Spectramax M2 Microplate Reader and the results were calculated using a standard graphic.

\section{Determination of TNF- $a$}

The Invitrogen Rat TNF-a ELISA kit was used in this measurement. Monoclonal TNF-a antibody recognizes and binds TNF-a found in the solution. Biotinylated polyclonal antibody recognizes the TNF-a bound protein composite. Streptavidin peroxidase recognizes the biotinylated complex even at the lowest concentrations of TNF-a in the presence of chromogene substrate. $50 \mu \mathrm{L}$ of the sample mixture was used in the experiment. Shortly, $50 \mu \mathrm{L}$ of the sample mixture or control mixture was incubated in the wells for 2 hours at room temperature before being washed for five times. After the fluid was completely washed away from the micro plates $50 \mu \mathrm{L}$ of biotinylated TNF- $a$ was added to the wells and incubated at room temperature for 2 hours. The wells were again washed 5 times to completely remove the remnant fluid. Then $50 \mu \mathrm{L}$ of streptavidin-peroxidase (HRP) was added and incubated for 30 minutes. Then the wells were washed again five times to remove all unbound enzyme. Afterwards, $50 \mu \mathrm{L}$ of chromogene substrate was added to each well and incubated for 8 minutes. Finally $50 \mu \mathrm{L}$ of $0.5 \mathrm{~N} \mathrm{HCl}$ Acid was added to stop the color formation reaction and the results were immediately evaluated using Molecular Devices Spectramax M2 Microplate reader at a wave length of $450 \mathrm{~nm}$. The results were calculated from a standard graphic derived from regression analysis consisting of 4 parameters.

\section{Determination of Interleukin-6}

Invitrogen Rat IL-6 ELISA kit was used in this measurement. Monoclonal IL-6antibody recognizes and binds IL-6 found in the solution. Biotinylated polyclonal antibody recognizes the IL-6 bound protein composite. Streptavidin peroxidase recognizes the biotinylated complex even at the lowest concentrations of IL-6 in the presence of chromogene substrate. $50 \mu \mathrm{L}$ of the sample mixture was used in the experiment. Shortly, $50 \mu \mathrm{L}$ of the sample mixture or control mixture was incubated in the wells for 2 hours at room temperature before being washed for five times. After the fluid was completely washed away from the micro plates, $50 \mu \mathrm{L}$ of biotinylated IL-6 was added to the wells and incubated at room temperature for 2 hours. The wells were again washed 5 times to completely remove the remnant fluid. Then $50 \mu \mathrm{L}$ of streptavidin-peroxidase (HRP) was added and incubated for 30 minutes. Then the wells 
were washed again five times to remove all unbound enzyme. Afterwards, $50 \mu \mathrm{L}$ of chromogene substrate was added to each well and incubated for 8 minutes. Finally $50 \mu \mathrm{L}$ of 0.5 $\mathrm{N} \mathrm{HCl}$ Acid was added to stop the color formation reaction and the results were immediately evaluated using Molecular Devices Spectramax M2 Microplate reader at a wave length of $450 \mathrm{~nm}$. The results were calculated from a standard graphic derived from regression analysis consisting of 4 parameters.

\section{Functional Recovery Assessment}

For motor function, animals were classified using a Modified Tarlov 5-point scale: grade $1=$ no voluntary hind limb movement; grade 2 = minimal voluntary hind limb movements but unable to stand; grade 3 = able to stand but unable to walk; grade $4=$ able to walk with mild spasticity or in coordination of the hind limbs and grade $5=$ able to walk normally (21).

\section{RESULTS}

\section{Lipid Peroxidation}

Lipid peroxidation levels are expressed in terms of nanomole per gram tissue over the MDA amount. After the analysis of the difference between 5 experimental groups with 1-way ANOVA test, significant difference has been established $(P=0.001)$. The a-LA administration at the dose of $100 \mathrm{mgr} / \mathrm{kg}$ significantly reduced the lipid peroxidation level of the LA group (0.87) with respect to the LT group, in which only trauma was introduced after laminectomy (1.85) $(\mathrm{P}=0.001)$. The NAC administration at the dose of $300 \mathrm{mgr} / \mathrm{kg}$ has significantly reduced the lipid peroxidation level of the NAC group (1.14) with respect to the LT group (1.85) $(P=0.001)$. For the investigation of the vehicle effect, the lipid peroxidation ratios of the LT and PEG groups (IP $0.5 \mathrm{~mL}$ PEG) were compared individually by post hoc analysis Dunnett T3 test, and no significant difference was found within these 2 groups $(\mathrm{P}=0.637)$. The overall results are summarized in Table II and Figure 1.

\section{TNF-a}

TNF-a levels are expressed in terms of pictograms per milliliter tissue. After the analysis of the difference between
5 experimental groups with the ANOVA variance test, a significant difference was established $(P=0.001)$. Post hoc analysis (TUKEY-LSD) was used to evaluate significant differences between the groups. The $a-L A$ administration at a dose of $100 \mathrm{mgr} / \mathrm{kg}$ significantly reduced the TNF-a levels of LA group (32.15) with respect to the LT group where only trauma was introduced after laminectomy (53.23) $(P=0.001)$. The NAC administration at a dose of $300 \mathrm{mgr} / \mathrm{kg}$ significantly reduced the TNF-a levels as well in the NAC group (33.9) with respect to the $L T$ group (53.23) $(P=0.001)$. For the investigation of the vehicle effect, the TNF-a levels of the LT and PEG groups (IP $0.5 \mathrm{~mL}$ PEG) were compared individually by post hoc analysis Dunnett T3 test, and no significant difference was found within these 2 groups $(P=0.411)$. The overall results are summarized in Table II and Figure 2.

\section{Interleukin-6}

IL-6 levels are expressed in terms of pictograms per mililiter tissue. After the analysis of the difference between 5 experimental groups with the ANOVA variance test, a significant difference was established $(P=0.001)$. Post hoc analysis (TUKEY-LSD) was used to evaluate significant differences in between groups. The a-LA administration at a dose of $100 \mathrm{mgr} / \mathrm{kg}$ significantly reduced the IL-6 levels of LA group (306.1) with respect to LT group where no treatment modality was used (560.27) $(\mathrm{P}=0.001)$. The NAC administration at a dose of $300 \mathrm{mgr} / \mathrm{kg}$ significantly reduced the IL-6 levels as well in the NAC group (372.51) with respect to the LT group (560.27) $(\mathrm{P}=0.001)$. For the investigation of vehicle effect, the IL-6 levels of the LT and PEG groups (IP $0.5 \mathrm{~mL}$ PEG) were compared individually by post hoc analysis Dunnett T3 test, and no significant difference was found within these 2 groups $(P=0.312)$. The overall results are summarized in Table II and Figure 3.

\section{Motor Function Findings}

At the day after trauma, the respective mean motor scores and their standard deviation in groups 1, 2, 3, 4 and 5 were $4.5714 \pm 0.53,1.125 \pm 0.31,3.375 \pm 0.44, \quad 3.125 \pm 0.83$ and $1.285 \pm 0.48$ (Table III). The Chi-Square test was used to

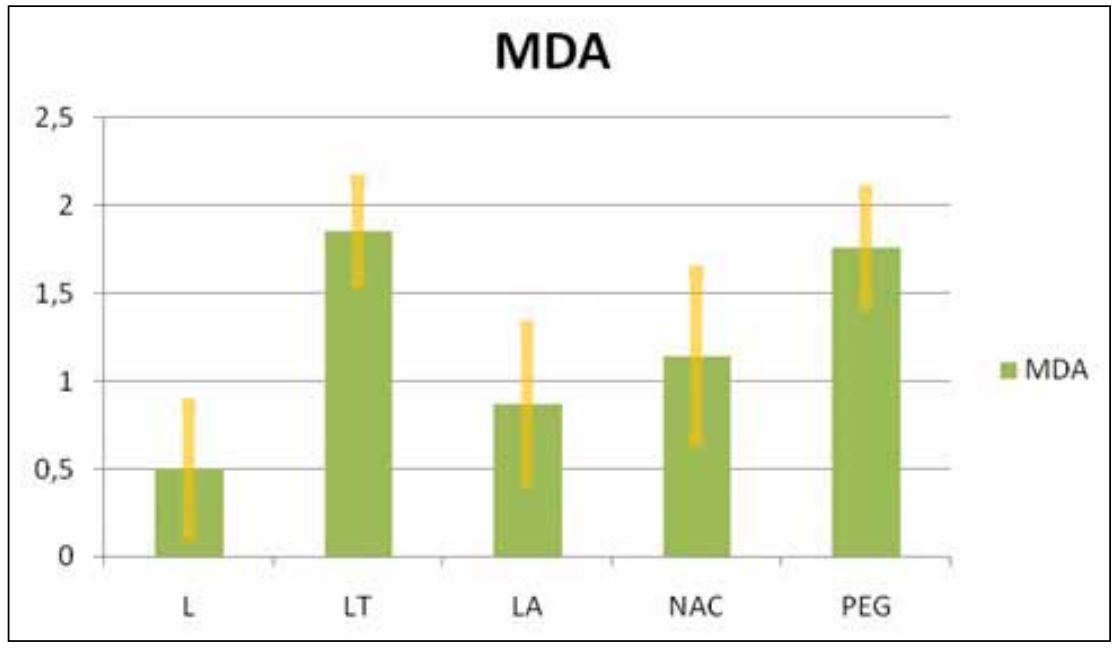

Figure 1: Mean MDA levels for each group along with standard deviation.

$\mathbf{L}=$ laminectomy

$\mathbf{L T}=$ laminectomy trauma

LA= laminectomy trauma $a-L A$

NAC= laminectomy trauma

NAC PEG= vehicle group $\operatorname{MDA}(\mu \mathrm{M} / \mathrm{mg})$. 


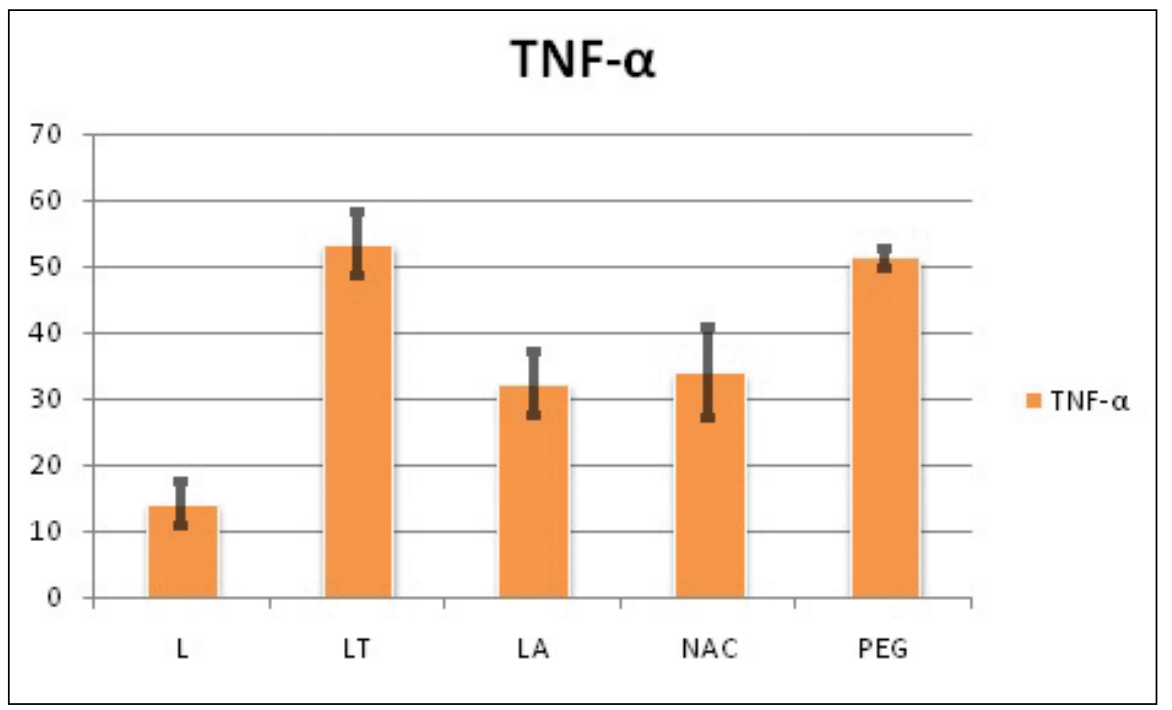

Figure 2: Mean TNF-a levels for each group along with standard deviation.

$\mathbf{L}=$ laminectomy

$\mathbf{L T}=$ laminectomy trauma

LA= laminectomy trauma a-LA

NAC= laminectomy trauma

NAC PEG= vehicle group

TNF-a (pg/ml).

Table II: Mean Values of MDA, TNF-a and IL-6 for Each Group and Their P Values in Comparison

\begin{tabular}{|c|c|c|c|c|c|c|c|c|c|c|}
\hline & & $\mathbf{n}$ & mean & SD* & $\mathbf{P}$ & $P_{\text {L-LT }}$ & $\mathbf{P}_{\text {LT-LA }}$ & $\mathbf{P}_{\text {LT-NAC }}$ & $\mathbf{P}_{\text {LT-PEG }}$ & $P_{\text {LA-NAC }}$ \\
\hline MDA & $\mathrm{L}$ & 7 & 0.5 & 0.37 & 0.001 & 0.001 & 0.001 & 0.001 & 0.637 & 0.139 \\
\hline \multirow[t]{4}{*}{$(\mu \mathrm{M} / \mathrm{mg})$} & LT & 8 & 1.85 & 0.29 & & & & & & \\
\hline & LA & 8 & 0.87 & 0.44 & & & & & & \\
\hline & NAC & 8 & 1.14 & 0.48 & & & & & & \\
\hline & PEG & 7 & 1.76 & 0.32 & & & & & & \\
\hline TNF-a & $\mathrm{L}$ & 7 & 14.02 & 3.23 & 0.017 & 0.001 & 0.001 & 0.001 & 0.411 & 0.467 \\
\hline \multirow[t]{4}{*}{$(\mathrm{pg} / \mathrm{ml})$} & $\mathrm{LT}$ & 8 & 53.23 & 4.81 & & & & & & \\
\hline & LA & 8 & 32.15 & 4.9 & & & & & & \\
\hline & NAC & 8 & 33.9 & 6.83 & & & & & & \\
\hline & PEG & 7 & 51.2 & 1.47 & & & & & & \\
\hline IL-6 & $\mathrm{L}$ & 7 & 99.82 & 9.14 & 0.012 & 0.001 & 0.001 & 0.001 & 0.312 & 0.194 \\
\hline \multirow[t]{4}{*}{ (pg/ml) } & LT & 8 & 560.27 & 133.1 & & & & & & \\
\hline & LA & 8 & 306.1 & 81.79 & & & & & & \\
\hline & NAC & 8 & 372.51 & 91.81 & & & & & & \\
\hline & PEG & 7 & 509.54 & 113.15 & & & & & & \\
\hline
\end{tabular}

*Standard Deviation. $P$ value $<0.005$ was accepted as significant.

Table III: Modified Tarlov Test Results of the Groups

\begin{tabular}{|c|c|c|c|c|c|c|c|c|c|}
\hline & Groups & $\mathbf{n}$ & Mean & SD* $^{*}$ & $P_{\text {L-LT }}$ & $\mathbf{P}_{\text {LT-LA }}$ & $P_{\text {LT-NAC }}$ & $\mathbf{P}_{\text {LT-PEG }}$ & $\mathbf{P}_{\text {LA-NAC }}$ \\
\hline \multirow[t]{5}{*}{ Tarlov } & $\mathrm{L}$ & 7 & 4.5714 & 0.53 & 0.001 & 0.001 & 0.001 & 0.621 & 0.427 \\
\hline & LT & 8 & 1.125 & 0.31 & & & & & \\
\hline & LA & 8 & 3.375 & 0.44 & & & & & \\
\hline & NAC & 8 & 3.125 & 0.83 & & & & & \\
\hline & PEG & 7 & 1.285 & 0.48 & & & & & \\
\hline
\end{tabular}

*Standard Deviation. $P$ value $<0.005$ was accepted as significant. 

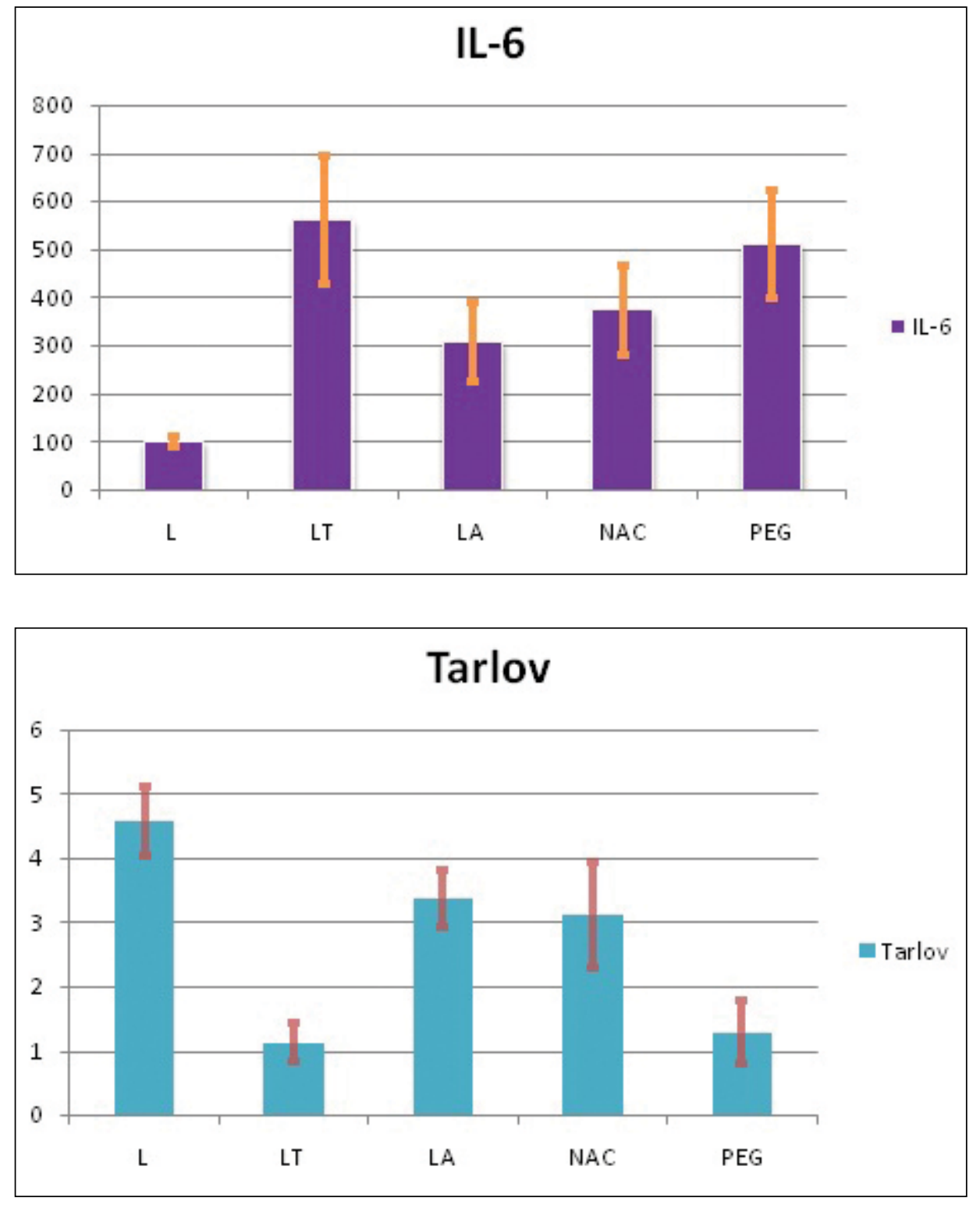

Figure 3: Mean IL-6 levels for each group along with standard deviation.

$\mathbf{L}=$ laminectomy

$\mathbf{L T}=$ laminectomy trauma

LA= laminectomy trauma a-LA

NAC= laminectomy trauma NAC

$\mathbf{P E G}=$ vehicle group

IL-6 (pg/ml).
Figure 4: Mean Modified Tarlov Scores for each group with SD. $\mathbf{L}=$ laminectomy $\mathbf{L T}=$ laminectomy trauma LA = laminectomy trauma $a-L A$ NAC = laminectomy trauma NAC PEG = vehicle group Modified Tarlov Scale (0-5). evaluate the results. There was an evident and statistically significant difference between groups L-LT, LT-LA and LT-NAC as seen in Table III and Figure $4(\mathrm{P}=0.001)$.

Overall, the parameters tested in this study demonstrate a positive effect in both the LA and NAC groups compared with the LT group. However, the statistical analysis of both the biochemical and motor function results revealed no statistically significant difference between the LA and NAC groups.

\section{DISCUSSION}

Traumatic injury of neural tissue has been a topic of interest amongst scientists for almost a century. Enormous funding has been made available to reverse the secondary injury effects of spinal cord injury. Many agents have been proven to counteract the devastating results. For the primary injury phase, no treatment modalities have been proven to have significant positive results against the injury so far. On the other hand, the latter and continuous secondary injury mechanisms of neurotrauma has been evaluated frequently and thoroughly although there is a long path ahead of us. It is doubtless that studying drug interactions to reduce free radical-induced oxidative damage reactions and membrane lipid peroxidation in preclinical traumatic injury models is an ongoing endeavor.

Mitochondria play a pivotal role in determining the spectrum of secondary injury after neurotrauma. Thus preserving the functions of mitochondria has been an excellent target for cell viability after trauma. In the secondary injury phase, excitotoxicity leads to reduction in membrane potentials of mitochondria, enhancing the overproduction of ROS by excessive calcium intake. The overproduction of ROS triggers oxidative damage, neuro-inflammation, apoptosis and cell death (18). 
Oxidative damage occurs when there is an imbalance between free radical production and the anti-oxidant capacity of the cell. Halliwell et al. stated that there is a tendency to neuronal architecture disruption due to secondary oxidative injury possibly because of the mostly polyunsaturated fatty acids in cellular membranes that would be the target of lipid peroxidation damage and the incapability of neurons to regenerate, with neural tissues having insufficient antioxidant enzymes for mopping the overflow of ROS only worsening the cascade (9). Also ROS are supposed to cause secondary damage after central nervous system (CNS) injury by contributing to the oxidative damage of proteins such as DNA and membrane phospholipids. It has been demonstrated that the levels of superoxide anion, hydrogen peroxide, hydroxyl radical, catalytic iron, nitric oxide and peroxynitrite, as well as the products of oxidation and nitration of proteins and membrane lipid peroxidation, all significantly increase following $\mathrm{SCl}$ (13).

In the mitochondria, oxidative alteration of critical proteins' thiol groups can impair the cell functions. Redox status of critical thiol groups in signaling molecules such as kinases and phosphatases often determine the initiation and progression of signaling cascades, which decide cell death versus survival pathways (11).

Neuro-inflammation is another secondary injury mechanism. In the trauma area the B lymphocytes and microglia cause damage by generating proinflammatory cytokines such as TNF-a, IL-1 $\beta$ and IL-6 (4).

a-LA is a naturally occurring co-factor for pyruvate dehydrogenase and a-ketoglutarate dehydrogenase that plays a role in the antioxidant system and is synthesized in diminutive amounts by some plants and animals. a-LA can be found readily in dietary sources such as liver, spinach, broccoli and tomatoes (16). In human beings, a-LA is synthesized in the mitochondria from octanoic acid. a-LA is soluble not only in water, but also in fats and thus it can cross the blood-brain barrier without trouble and stabilize the blood-brain barrier ( 3 , 20).

NAC is a derivative of cysteine and has been clinically used as a mucolytic agent and for the treatment of acetaminophen intoxication. It acts as a glutathione (GSH) precursor and ameliorates pro-inflammatory cytokines (10).

Lipid peroxidation and inflammatory processes have been shown to take parts in cellular damage after trauma. In trauma, free radicals and iron compounds catalyze not only the generation of hydroxyl radical and cause lipid peroxidation but also increases pro-inflammatory cytokines such as TNF-a, IL-1 $\beta$ and IL-6 (22). The results in this study clearly confirm that trauma increases the levels of MDA, TNF- $a$ and IL- 6 in the spinal cord of rats at 24 hours after injury. Inhibition of neuro- inflammation and increasing the antioxidant capacity by administration of a-LA or NAC immediately after trauma both decrease lipid peroxidation levels and proinflammatory cytokines.

It could be hypothesized that the one of the effective approaches to interrupt secondary oxidative and pro-inflammatory damage after traumatic injury involves the antioxidants. In this study, it has been shown that the systemic administration of a-LA and NAC, just after spinal cord injury decreases levels of tissue lipid peroxidation and pro-inflammatory cytokines. The experimental results of this study correlate with the previous articles about the neuroprotective effects of $a-L A$ and NAC.

Before the research, it was hypothesized that a-LA would provide better outcomes both clinically and biochemically due to its lipophilic properties allowing it to cross the cell membrane more efficiently than NAC. However, although the statistical analysis reveals significant improvement with the use of both agents, no significant difference was established between the agents. $a-L A$ can easily pass through the bloodbrain barrier and also cellular membranes. On the other hand, NAC has restricted bioavailability because of low lipophilic properties and the negatively-charged carboxyl group. Since concentrations of a-LA are higher in the mitochondria, it was postulated that redox reactions could be induced more readily when compared with NAC. The literature review also turned up results where researchers recently emphasized the neuroprotective efficiency of NAC by using the amide derivative of NAC, which has a neutralized carboxylic group. The amide derivative of NAC becomes more lipophilic and willingly crosses blood brain barrier and cellular membranes as well $(15,18)$.

In conclusion, it is the suggestion of the authors of this paper that administration of antioxidant agents - $\mathrm{a}$-LA and NAC could ameliorate some of the secondary injury cascades even when administered just after spinal cord trauma. The defensive efficiency of a-LA against secondary injury cascades after traumatic spinal cord injury, indicated by this study, might be useful to encourage the clinical use of this antioxidant agent as a supportive treatment after spinal cord injury. More subjects with varying dosages and timing may reveal further compelling results regarding the efficiency of these and other agents. As time progresses, new agents against $\mathrm{SCl}$ could be exposed or more effective agents could be synthesized from previously well-tried agents.

\section{REFERENCES}

1. Akpinar D, Yargicoglu $P$, Derin N, Alicigüzel $Y$, Sahin $M$, Agar $A$ : The effect of lipoic acid on lipid peroxidation and visual evoked potentials (VEPs) in rats exposed to chronic restraint stress. Int $\mathrm{J}$ Neurosci 117: 1691-1706, 2007

2. Allen AR: Remarks on the histopathological changes in the spinal cord due to impact. An experimental study. J Nerv Ment Dis 41:141-147, 1914

3. Cakatay U, Kayali R: An overdose of alpha lipoic acid may cause trace element deficiency in diabetes mellitus. Med Hypotheses 67(3): 672-673, 2006

4. Donnelly DJ, Popovich PG: Inflammation and its role in neuroprotection, axonal regeneration and functional recovery after spinal cord injury. Exp Neurol 209:378-388, 2008

5. Floyd RA, West M, Hensley K: Oxidative biochemical markers; Clue to understanding aging in long-lived species. Exp Gerontol 36: 619-640, 2001

6. Furlan JC, Sakakibara BM, Miller WC, Krassioukov AV: Global incidence and prevalence of traumatic spinal cord injury. Can J Neurol Sci 40(4):456-464, 2013 
7. Gorąca A, Asłanowicz-Antkowiak K: Prophylaxis with a-lipoic acid against lipopolysaccharide-induced brain injury in rats. Archivum Immunologiae et Therapiae Experimentalis 57(2):141-146 2009

8. Haber M, Abdel Baki SG, Grin'kina NM, Irizarry R, Ershova A, Orsi $\mathrm{S}$, Grill RJ, Dash P, Bergold PJ: Minocycline plus N-acetylcysteine synergize to modulate inflammation and prevent cognitive and memory deficits in a rat model of mild traumatic brain injury. Exp Neurol 249:169-177, 2013

9. Halliwell B: Oxidative stress and neurodegeneration: Where are we now? J Neurochem 97: 1634-1658, 2006

10. Karalija A, Novikova LN, Kingham PJ, Wiberg M, Novikov LN: The effects of $\mathrm{N}$-acetyl-cysteine and acetyl-L-carnitine on neural survival, neuroinflammation and regeneration following spinal cord injury. Neuroscience 269:143-151, 2014

11. Karunakaran S, Diwakar L, Saeed U, Agarwal V, Ramakrishnan $\mathrm{S}$, lyengar S, Ravindranath V: Activation of apoptosis signal regulating kinase 1 (ASK1) and translocation of death-associated protein, Daxx, in substantia nigra pars compacta in a mouse model of Parkinson's disease: Protection by alpha-lipoic acid. FASEB J 21(9): 2226-2236, 2007

12. Laskin DL, Pendino KJ: Macrophages and inflammatory mediators in tissue injury. Annu Rev Pharmacol Toxicol 35: 655-677, 1995

13. Ling X, Bao F, Qian H, Liu D: The temporal and spatial profiles of cell loss following experimental spinal cord injury: Effect of antioxidant therapy on cell death and functional recovery. BMC Neurosci 14:146, 2013

14. Maxwell RE, Long DM, French LA: The effects of glucosteroids on experimental cold induced brain edema. Gross morphological alterations and vascular permeability changes. J Neurosurg 34 : 477-487, 1971
15. Patel SP, Sullivan PG, Pandya JD, Goldstein GA, VanRooyen $\mathrm{JL}$, Yonutas HM, Eldahan KC, Morehouse J, Magnuson DS, Rabchevsky AG: $\mathrm{N}$-acetylcysteine amide preserves mitochondrial bioenergetics and improves functional recovery following spinal trauma. Exp Neurol 257:95-105, 2014

16. Perera J, Tan JH, Jeevathayaparan S, Chakravarthi S, Haleagrahara N: Neuroprotective effects of alpha lipoic Acid on haloperidol-induced oxidative stress in the rat brain. Cell Biosci 1(1):12, 2011

17. Reed LJ, DeBusk BG, Gunsalus IC, Hornberger CS Jr: Crystalline alpha-lipoic acid; a catalytic agent associated with pyruvate dehydrogenase. Science 114:93-94, 1951

18. Semple BD: Early preservation of mitochondrial bioenergetics supports both structural and functional recovery after neurotrauma. Exp Neurol 261:291-297, 2014

19. Senturk S, Gurcay AG, Bozkurt I, Gurcan O, Eroglu H, Turkoglu OF, Bodur E, Bavbek M: Effects of tadalafil-Type-V phosphodiesterase enzyme inhibitor-On rats with spinal trauma. Br J Neurosurg 7:16, 2014

20. Skibska B, Józefowicz-Okonkwo G, Goraca A: Protective effects of early administration of alpha-lipoic acid against lipopolysaccharide-induced plasma lipid peroxidation. Pharmacol Rep 58(3):399-404, 2006

21. Tarlov IM, Klinger H: Spinal cord compression studies: III. Time 1. limits for recovery after acute compression in dogs. AMA Arch Neurol Psychiatry 71:588 - 597, 1954

22. Turkoglu OF, Eroglu H, Okutan O, Gurcan O, Bodur E, Sargon MF, Oner L, Beskonakli E: Atorvastatin efficiency after traumatic brain injury in rats. Surg Neurol 72(2): 146-152, 2009 